March 1994

QMW-TH-94/11

SUSX-TH-94/11

\title{
Anisotropic Solutions For Orbifold Moduli From Duality Invariant Gaugino Condensates.
}

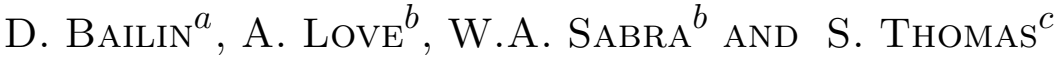 \\ a School of Mathematical and Physical Sciences, \\ University of Sussex, Brighton BN1 9QH, U.K. \\ ${ }^{b}$ Department of Physics, \\ Royal Holloway and Bedford New College, \\ University of London, \\ Egham, Surrey, U.K. \\ ${ }^{c}$ Department of Physics, \\ Queen Mary and Westfield College, \\ University of London, \\ Mile End Road, London, U.K.
}

\begin{abstract}
The values of the $T$ and $U$ moduli are studied for those $\mathbf{Z}_{N}$ Coxeter orbifolds with the property that some of the twisted sectors have fixed planes for which the six-torus $\mathbf{T}^{6}$ can not be decomposed into a direct sum $\mathbf{T}^{2} \bigoplus \mathbf{T}^{4}$ with the fixed plane lying in $\mathbf{T}^{2}$. Such moduli in general transform under a subgroup of the modular group $S L(2, Z)$. The moduli are determined by minimizing the effective potential derived from a duality invariant gaugino condensate.
\end{abstract}


The values of the untwisted $T$ and $U$ moduli for an orbifold are of importance because they enter the string loop threshold corrections relevant to unification of gauge coupling constants [1-4] and the soft supersymmetry breaking terms derived from orbifold compactifications [6]. The effective potential is flat in these moduli to all orders of string perturbation theory and some non-perturbative mechanism is therefore required to provide an effective potential that can determine the moduli. A promising mechanism is a non-perturbative superpotential due to gaugino condensation in the hidden sector [5]. This approach has been applied to the calculation of the moduli by incorporating target-space duality invariance [7-13]. The derivation of this non-perturbative superpotential requires a knowledge of the moduli dependence of the string loop threshold corrections to gauge coupling constants. This moduli dependence is associated [1] with orbifold twisted sectors for which there is a complex plane of the six torus $\mathbf{T}^{6}$ fixed by the twists in both space and time worldsheet directions. The threshold corrections take the standard form [1-4] with $S L(2, Z)$ modular symmetry group when for all such fixed planes, $\mathbf{T}^{6}$ can be decomposed into a direct sum $\mathbf{T}^{2} \oplus \mathbf{T}^{4}$ with the fixed plane lying in $\mathbf{T}^{2}$. The moduli dependent threshold corrections have also been calculated for all $\mathbf{Z}_{N}$ Coxeter orbifolds for which this simplifying assumption does not apply [14, 15]. We shall refer to these as non-decomposable orbifolds. For non-decomposable orbifolds the threshold corrections have modified forms with modular symmetry groups [14-16] which are subgroups of $S L(2, Z)$, not always the full group. It is our purpose here, to study the values of the moduli for such non-decomposable $\mathbf{Z}_{N}$ orbifolds. The lattices for which $\mathbf{Z}_{N}$ point groups provide such orbifolds are listed in Table 1.

Previous work [7-13] on minimizing the effective potential to determine the moduli has been confined, for convenience, to the isotropic case where the three generic moduli $T_{i}, i=1,2,3$, have been set all equal so that there is a single overall moduli $T$. For the non-decomposable orbifolds discussed, this is no longer an appropriate assumption because the string loop threshold corrections place $T_{1}$, $T_{2}$ and $T_{3}$ on a different footing. We shall also include the contribution of the 
$U$-moduli to the effective potential which has previously been neglected. However, we shall confine our attention to the pure gauge case without matter fields coupling to the hidden sector gauge group. Since we are dealing with modular symmetry groups that are subgroups of $S L(2, Z)$, duality anomaly cancellation considerations continue to lead to a Kahler potential $K$ of the form

$$
K=-\ln y-\sum_{i} \ln \left(T_{i}+\bar{T}_{i}\right)-\sum_{m} \ln \left(U_{m}+\bar{U}_{m}\right)
$$

where

$$
y=S+\bar{S}-\sum_{i} \frac{\delta_{\mathrm{GS}}^{i}}{8 \pi^{2}} \ln \left(T_{i}+\bar{T}_{i}\right)-\sum_{m} \frac{\tilde{\delta}_{\mathrm{GS}}^{m}}{8 \pi^{2}} \ln \left(U_{m}+\bar{U}_{m}\right) .
$$

In (1) and (2), $S$ is the dilaton field, the sum over $i$ runs from 1 to 3 and the sum over $m$ from 1 to the hodge number $h_{(2,1)}$. Off-diagonal moduli, $T_{i j}$ which are present for some $\mathbf{Z}_{N}$ orbifolds, but never contribute to string loop threshold corrections have been dropped. The Green-Schwarz coefficients $\delta_{\mathrm{GS}}^{i}$ and $\tilde{\delta}_{\mathrm{GS}}^{m}$ are required $[2,3]$ for the cancelation of the anomalies of the underlying $\sigma$-model. In this case, in order for the Kahler potential to transform as at tree level under modular transformations, one should allow a non-trivial modular transformation of the dilaton field $S$. In what follows it will usually be convenient for notational purposes to treat the $U$ moduli as additional $T$ moduli rather than to display them separately. In general, the effective potential $V$ is given by [7-13]

$$
\begin{aligned}
y \prod_{i}\left(T_{i}+\bar{T}_{i}\right) V & =\left|W-y \frac{\partial W}{\partial S}\right|^{2}-3|W|^{2} \\
& +\sum_{i} \frac{y}{\left(y-\frac{\delta_{\mathrm{GS}}^{i}}{8 \pi^{2}}\right)}\left|W-\frac{\delta_{\mathrm{GS}}^{i}}{8 \pi^{2}} \frac{\partial W}{\partial S}-\left(T_{i}+\bar{T}_{i}\right) \frac{\partial W}{\partial T_{i}}\right|^{2}
\end{aligned}
$$

where $W$ is the superpotential and the sums and products over $i$ are now understood to include the $U$ moduli. 
To be able to fix the values of the moduli, the modular invariant gaugino condensate superpotential is required. This in turn, requires a knowledge of the string loop threshold corrections to the gauge coupling constants. In general, for the factor $G_{a}$ of the hidden sector gauge group, the running coupling constant $g_{a}(\mu)$ obeys

$$
g_{a}^{-2}(\mu)=g_{a}^{-2}(M)+\frac{b_{a}}{16 \pi^{2}} \ln \left(\frac{M^{2}}{\mu^{2}}\right)+\frac{\triangle_{a}}{16 \pi^{2}},
$$

where $M$ is the string scale and $\triangle_{a}$ is the threshold correction. For the nondecomposable $\mathbf{Z}_{N}$ orbifolds (in the sense discussed above), $\triangle_{a}$ takes the general form $[14,15]$

$$
\triangle_{a}=-\sum_{i}\left({b^{\prime}}_{a}^{i}-\delta_{\mathrm{GS}}^{i}\right)\left(\ln \left(T_{i}+\bar{T}_{i}\right)+\sum_{m} \frac{C_{i m}}{2} \ln \left|\eta\left(\frac{T_{i}}{l_{i m}}\right)\right|^{4}\right)
$$

where the range over which $m$ runs depends on the value of $i$,

$$
\sum_{m} C_{i m}=2
$$

for all $i$. The coefficients $C_{i m}$ and $l_{i m}$ are given in Table 2, for the various nondecomposable Coxeter orbifolds. The ${b^{\prime}}_{a}^{i}$ are the usual coefficients [2, 3] which are determined by the contribution of the massless states to the duality anomaly in a way which does not depend on the underlying lattice for the orbifold,

$$
\begin{aligned}
& {b^{\prime}}_{a}^{i}=-C\left(G_{a}\right)+\sum_{R_{a}} T\left(R_{a}\right)\left(1+2 n_{R_{a}}^{i}\right), \quad \text { for } T \text { moduli } \\
& {b^{\prime}}_{a}^{i}=-C\left(G_{a}\right)+\sum_{R_{a}} T\left(R_{a}\right)\left(1+2 l_{R_{a}}^{i}\right), \quad \text { for } U \text { moduli. }
\end{aligned}
$$

In (7), $C\left(G_{a}\right)$ and $T\left(R_{a}\right)$ are Casimirs for the gauge group factor $G_{a}$ and its representations $R_{a}, n_{R_{a}}^{i}$ and $l_{R_{a}}^{i}, i=1,2,3$ are the modular weights under $T$ and $U$ duality transformation respectively, for the massless states in the representation 
$R_{a}$ of $G_{a}$. For the pure gauge case, where there is no matter charged under the hidden sector gauge group, (7) simplifies to

$$
{b^{\prime}}_{a}^{i}=-C\left(G_{a}\right)=\frac{b_{a}}{3} .
$$

The holomorphic part $f_{a}^{W}$ of the gauge kinetic function is given by

$$
f_{a}^{W}=S-\sum_{i, m} \frac{\left(b^{\prime}{ }_{a}^{i}-\delta_{\mathrm{GS}}^{i}\right)}{8 \pi^{2}} C_{i m} \ln \left(\eta\left(\frac{T_{i}}{l_{i m}}\right)\right)
$$

with $S$ the dilaton field whose real part $S_{R}$ controls the value of the gauge coupling constant at the string unification scale*

The modular invariant superpotential $W_{n p}$ is of the form $[7,12,17,9]$

$$
W_{n p}=\sum_{a} W_{n p}^{a}=\sum_{a} d_{a} e^{\frac{24 \pi^{2}}{b_{a}} f_{a}^{W}}
$$

and (9) gives

$$
W_{n p}^{a}=d_{a} e^{\frac{24 \pi^{2}}{b_{a}} S} \prod_{i, m}\left(\eta\left(\frac{T_{i}}{l_{i m}}\right)\right)^{-C_{i m}\left(1-\frac{3 \delta_{\mathrm{GS}}^{i}}{b_{a}}\right)}
$$

Notice that (6), together with the non-trivial modular transformation of the dilaton field $S$ beyond the tree level $[2,3]$, ensures that $W_{n p}^{a}$ has the correct modular weight of -1 for modular transformations on each of the $T_{i}$ separately.

When there is at least one complex plane that is rotated by all non-trivial powers of the point group generator $\theta$, i.e., at least one $N=1$ complex plane, then the fact that such a plane does not contribute to the moduli dependent threshold

\footnotetext{
* Strictly speaking, when $\delta_{\mathrm{GS}}^{i}$ and $\tilde{\delta}_{\mathrm{GS}}^{m} \neq 0$, it is the $y$ field given in (2) which takes on this role.
} 
corrections implies, [13]

$$
{b^{\prime}}_{a}^{i}=\frac{b_{a}}{3}=\delta_{\mathrm{GS}}^{i}
$$

so that $b_{a}$ is independent of $a$,

$$
b_{a}=b, \quad \text { for all } a .
$$

In these circumstances, each of the $W_{n p}^{a}$ has the same $e^{24 \pi^{2} S / b}$ dependence on the dilaton field and there is effectively a single gaugino condensate. When $W=W_{n p}$ is substituted in (3), this results in there being no minimum for $S_{R}$ and the value of the dilaton field not being determined. We shall assume with previous authors [7, 12 ] that some unspecified mechanism fixes the dilaton field at a value in the region of weak coupling. Following [13], we shall take $S_{R} \approx 2$. (One mechanism that has been discussed in the literature is for there to be charged matter $[9,13,17]$ coupled to the hidden sector gauge group with more than one condensate $[8,9,18]$. An intermediate scale mass for this matter can be generated by the expectation values of certain singlet scalars, which can be 'integrated out' by minimizing the effective potential. It can then sometimes happen that there is a realistic minimum for the dilaton expectation value and that the $T_{i}$ dependence of the effective potential is the same as for the pure gauge case at least for $\delta_{\mathrm{GS}}^{i}=0$. Similar calculations can be carried out here, with the same outcome. However, the particular mechanism for fixing $S_{R}$ can not work for $\mathbf{Z}_{N}$ orbifolds because there is always at least one $N=1$ complex plane and, consequently, effectively a single gaugino condensate, even in the presence of matter, because of the requirement of correct modular weight for the gaugino condensate superpotential.)

Once the dilaton expectation value $S_{R}$ has been fixed at a value in the region of weak coupling, the effective potential (3) with $W=W_{n p}$ given by (10) and (11) may be minimized to find the values of the moduli $T_{i}$ (including the $U$ moduli.) The $N=$ 1 moduli will be exceptions to this procedure, since they do not enter the threshold correction formula (5). We will discuss their role more fully later. Because of 
the invariance of the effective potential under modular transformations on each modulus separately there will, generally speaking, be extrema at the fixed points of modular transformations (the self-dual points.) For the case of an $S L(2, Z)$ transformation

$$
T_{i} \rightarrow \frac{a_{i} T_{i}-i b_{i}}{i c_{i} T_{i}+d_{i}}
$$

where $a_{i}, b_{i}, c_{i}$ and $d_{i}$ are integers with $a_{i} d_{i}-b_{i} c_{i}=1$. The fixed points [7] are at $\tilde{T}_{i}=i$ or $e^{\pi i / 3}$ where $\tilde{T}_{i}=i T_{i}$ and they are fixed by the modular transformations $\mathcal{S}$ and $\mathcal{T} \mathcal{S}$, respectively, where

$$
\begin{array}{ll}
\mathcal{S}: & \tilde{T} \rightarrow-\frac{1}{\tilde{T}}, \\
\mathcal{T}: & \tilde{T} \rightarrow \tilde{T}+1 .
\end{array}
$$

Here, for non-decomposable $\mathbf{Z}_{N}$ orbifolds, the relevant modular symmetries for the $T$ and $U$ moduli [14-16] deriving from the string loop threshold corrections are in general congruence subgroups of $S L(2, Z)$. The congruence subgroups that arise are $\Gamma_{0}(2), \Gamma_{0}(3), \Gamma^{0}(2)$ and $\Gamma^{0}(3)$ where $\Gamma_{0}(n)$ and $\Gamma^{0}(n)$ are represented by the matrices

$$
\Gamma_{0}(n) \quad:\left(\begin{array}{ll}
a & b \\
c & d
\end{array}\right), \quad a d-b c=1, \quad c=0(\bmod n)
$$

and

$$
\Gamma^{0}(n) \quad:\left(\begin{array}{ll}
a & b \\
c & d
\end{array}\right), \quad a d-b c=1, \quad b=0(\bmod n)
$$

The corresponding fundamental domains are

$$
\begin{array}{ll}
\Gamma_{0}(2): & \mathcal{F}_{1}=\{\mathcal{I}, \mathcal{S}, \mathcal{S} \mathcal{T}\} \mathcal{F}, \\
\Gamma_{0}(3): & \mathcal{F}_{2}=\left\{\mathcal{I}, \mathcal{S}, \mathcal{S} \mathcal{T}, \mathcal{S} \mathcal{T}^{2}\right\} \mathcal{F}, \\
\Gamma^{0}(2): & \mathcal{F}_{3}=\{\mathcal{I}, \mathcal{S}, \mathcal{T} \mathcal{S}\} \mathcal{F}, \\
\Gamma^{0}(3): & \mathcal{F}_{4}=\left\{\mathcal{I}, \mathcal{S}, \mathcal{T} \mathcal{S}, \mathcal{T}^{2} \mathcal{S}\right\} \mathcal{F}
\end{array}
$$


where

$$
\mathcal{F}=\left\{\tilde{T}: \operatorname{Im} \tilde{T}>0, \quad|\operatorname{Re} \tilde{T}|<\frac{1}{2}, \quad|\tilde{T}|>1\right\}
$$

is the usual fundamental domain for $S L(2, Z)$. The fixed points for the congruence subgroups can now be found from the fixed points of $S L(2, Z)$ by noticing that if $z$ is a fixed point of the transformation $L$ lying in $\alpha \mathcal{F}$, with $\alpha=\mathcal{S}, \mathcal{S T}, \mathcal{T S}, \mathcal{S T}^{2}$ and $\mathcal{T}^{2} \mathcal{S}$, then $\alpha^{-1} z$ is a fixed point of $\alpha^{-1} L \alpha$ lying in $\mathcal{F}$, with the outcome that the possible fixed points are $T_{i}=\frac{1+i}{2}, T_{i}=1-i$ for $\Gamma_{0}(2)$ and $\Gamma^{0}(2)$ respectively, $T_{i}=\frac{1+i \sqrt{3}}{2 \sqrt{3}}$ for $\Gamma_{0}(3)$ and $T_{i}=\frac{\sqrt{3}}{2}(1-i \sqrt{3})$ for $\Gamma^{0}(3)$. These points are fixed by the elements $\mathcal{S} \mathcal{S}(\mathcal{S T})^{-1} \in \Gamma_{0}(2), \mathcal{T S} \mathcal{T}^{-1} \in \Gamma^{0}(2), \mathcal{S} \mathcal{T}^{2} \mathcal{S}(\mathcal{S T})^{-1} \in \Gamma_{0}(3)$ and $\mathcal{T}^{2} \mathcal{S} \mathcal{T}^{-1} \in \Gamma^{0}(3)$. In the $S L(2, Z)$ case with a single overall modulus [7], and $\delta_{\mathrm{GS}}^{i}=0$, the extrema associated with the two fixed points in the fundamental domain $\mathcal{F}, T=1, e^{-\pi i / 6}$ are a saddle point and a maximum respectively, with the minimum lying close to them. However as noted in $[7,12]$, the exact position of the minima in $T$ relative to the fixed points depends on the value of $S_{R}$. For the case of the subgroups, we see that there is a single fixed point in the corresponding fundamental region. The connection between this fixed point and extrema of $V$ is rather more involved since unlike [7], we do not assume that all the $\delta_{\mathrm{GS}}^{i}$ are necessarily vanishing. We will return to this issue later.

To carry out the minimization of the effective potential (3) to determine the true minima, values of $\delta_{\mathrm{GS}}^{i}$ are required. These may be determined for any particular orbifold by a comparison of the expression given in ref. [2] for the string loop threshold corrections in terms of ${b^{\prime}}^{i}$ and $\delta_{\text {GS }}^{i}$ with the expression given in [1] in terms of the renormalization group equation coefficients $\left(b_{a}^{N=2}\right)^{i}$ for the $N=2$ orbifold $\mathbf{T}^{6} / G_{i}$ where $G_{i}$ is the subgroup of the point group $G$ that leaves the $i$-th complex plane unrotated. Thus,

$$
\delta_{\mathrm{GS}}^{i}=b_{a}^{\prime i}-\left(b_{a}^{N=2}\right)^{i} \frac{\left|G_{i}\right|}{|G|} .
$$

This expression applies equally for $T$ and $U$ moduli provided we use the appropriate modular weights [6] in the calculation of ${b^{\prime}}_{a}^{i}$. In the case of a pure gauge hidden 
sector

$$
\delta_{\mathrm{GS}}^{i}=\frac{b_{a}}{3}\left(1-2 \frac{\left|G_{i}\right|}{|G|}\right) .
$$

For the $\mathbf{Z}_{N}$ orbifolds considered here (and with $b=-90$ for $E_{8}$ )

$$
\begin{array}{rrl}
\delta_{\mathrm{GS}}^{3}=0, & Z_{4}, \quad Z_{8}-I I \\
\delta_{\mathrm{GS}}^{1}=-10, \quad \delta_{\mathrm{GS}}^{3}=0, & Z_{6}-I I \\
\delta_{\mathrm{GS}}^{3}=-10, & Z_{12}-I .
\end{array}
$$

For moduli associated with $N=1$ planes, $\delta_{\text {GS }}^{i}$ is given by (12). Whilst the task of minimizing the potential (3) in the $T$ and $U$ moduli is best carried out numerically, it is instructive to calculate $\partial_{T_{i}} V$ and $\partial_{S} V$, in order to see the connection between fixed points of the modular (sub)groups discussed above, and certain extrema of $V$. One finds that the extrema conditions for the $T_{i}$ and $S$ moduli are

$$
\begin{aligned}
\left(\frac{3 \delta_{\mathrm{GS}}^{i}}{b}-\right. & x) \sum_{j} \frac{x}{\left(x-\frac{3 \delta_{\mathrm{GS}}^{j}}{b}\right)}\left|\tilde{G}^{j}\right|^{2}+x\left(T_{i}+\bar{T}_{i}\right) 3 \delta_{\mathrm{GS}}^{i} \sum_{j}\left\{\frac{3 \delta_{\mathrm{GS}}^{j}}{\left(b x-3 \delta_{\mathrm{GS}}^{j}\right)^{2}} \frac{\left|\tilde{G}^{j}\right|^{2}}{\left(T_{i}+\bar{T}_{i}\right)}\right. \\
+ & \frac{x}{\left(x-\frac{3 \delta_{\mathrm{GS}}^{j}}{b}\right)}\left[\left(\frac{W_{T_{i}}}{W}\left(1-\frac{3 \delta_{\mathrm{GS}}^{j}}{b}\right)-\delta_{i j} \frac{W_{T_{j}}}{W}-\left(T_{j}+\bar{T}_{j}\right) \frac{W_{T_{i} T_{j}}}{W} \overline{\tilde{G}}^{j}\right.\right. \\
- & \left.\left.\tilde{G}^{j}\left(\frac{\bar{W}_{T_{j}}}{\bar{W}}\right) \delta_{i j}\right]\right\}-x \tilde{G}^{i}\left(x^{2}-2 x-2\right)+\frac{3 \delta_{\mathrm{GS}}^{i}}{b}(1-x)\left(x^{2}-2\right)=0
\end{aligned}
$$

and

$$
\left(2-x^{2}\right)(1-x)+\sum_{i}\left(\frac{x}{\left(x-\frac{3 \delta_{\mathrm{GS}}^{i}}{b}\right)}\right)^{2}\left[\left|\tilde{G}^{i}\right|^{2}\left(x-\frac{3 \delta_{\mathrm{GS}}^{i}}{b}-1\right)\right]=0
$$

where in (23) and (24), $x=\left(24 \pi^{2} / b\right) y$ and the functions $\tilde{G}^{j}$ are defined as

$$
\tilde{G}^{j}=\left(1-\frac{3 \delta_{\mathrm{GS}}^{j}}{b}\right)\left(T_{j}+\bar{T}_{j}\right) \hat{G}^{j}
$$


with

$$
\hat{G}^{j}=\left(\frac{1}{T_{j}+\bar{T}_{j}}+\sum_{m} C_{j m} \frac{\partial_{T_{j}} \eta\left(\frac{T_{j}}{l_{j m}}\right)}{\eta\left(\frac{T_{j}}{l_{j m}}\right)}\right)=\frac{1}{T_{j}+\bar{T}_{j}}+G_{2}^{j}
$$

In (26), $G_{2}^{j}$ are weight 2 Eisenstein functions for the corresponding modular subgroups discussed above. Since $\hat{G}^{j} \sim(\partial \triangle) /\left(\partial T_{j}\right)$, where $\triangle$ is the threshold correction (5) for a simple gauge group, it is clear that $\hat{G}^{j}$ vanish at the fixed points of the corresponding modular subgroups. $\left(\hat{G}^{j}\right.$ are the generalization to subgroups of $S L(2, Z)$, of the weight 2 modular functions transforming under the full $S L(2, Z)$ group described in [7].) It is also clear from (23) that such fixed points will be extrema of $V$ provided $\delta_{\mathrm{GS}}^{j}=0$ and (or) $x$ is restricted to its extremum value. We could have arrived at the same conclusion by symmetry considerations alone. If $V(S, T)$ is invariant under a modular (sub)group in which $T_{i}$ transform as in (14) then recalling that for $\delta_{\mathrm{GS}}^{i} \neq 0, S$ itself transforms as

$$
S \rightarrow S-\sum_{i} \frac{\delta_{\mathrm{GS}}^{i}}{8 \pi^{2}} \ln \left(i c_{i} T_{i}+d_{i}\right)=S^{\prime}
$$

one has

$$
\frac{\partial V(S, T)}{\partial T_{i}}=\frac{1}{\left(i c_{i} T_{i}+d_{i}\right)^{2}} \frac{\partial V\left(S^{\prime}, T^{\prime}\right)}{\partial T_{i}^{\prime}}-\frac{i c_{i} \delta_{\mathrm{GS}}^{i}}{8 \pi^{2}\left(i c_{i} T_{i}+d_{i}\right)} \frac{\partial V\left(S^{\prime}, T_{i}^{\prime}\right)}{\partial S^{\prime}}
$$

Using the fact that $\left|i c_{i} T_{i}+d_{i}\right|=1, i c_{i} T_{i}+d_{i} \neq \pm 1$ at fixed points, we arrive at the above conditions for such points to be extrema. (Note that for fixed points, $S^{\prime}=S$ up to axionic shifts, which leave $V$ invariant.)

As stated earlier, the position we adopt in this paper is to fix the value of $S_{R} \approx 2$, which will not be an extremum of the potential $V$ ( at least not in the pure gauge case.) As a result, the connection between fixed points of modular subgroups and extrema of $V$ will not exist for those moduli having non-vanishing GreenSchwarz coefficients. We will now consider a specific example which will make these points clear and illustrate the differences between effective potentials that 
are invariant under modular subgroups of $S L(2, Z)$ rather than the full $S L(2, Z)$ as considered in [7]. An interesting example is that of the $Z_{6}-I I-b$ Coxeter orbifold. There are 3 moduli $T_{i}, i=1,2,3$, and a modulus $U_{3}$ which enter the effective potential, where the subscript refers to the corresponding complex plane. As demonstrated in $[15,16]$, the corresponding threshold corrections are invariant under $\Gamma_{T_{3}}^{0}(3), \Gamma_{U_{3}-2 i}^{0}(3)$, and $S L_{T_{1}}(2, Z)$ respectively, whilst $T_{2}$ being a modulus associated with an $N=1$ plane, does not contribute to the threshold corrections. The symmetry of the threshold correction transfers itself to that of $V$, even for $U_{3}-2 i$ because the Kahler potential only depends on Re $\left(U_{3}\right)$. However, note that the symmetry of $V$ with respect $U_{3}$ is not $\Gamma^{0}(3)$ but a different subgroup. This subgroup was identified in [15] as being

$$
U_{3} \rightarrow \frac{a U_{3}-i b}{i c U_{3}+d}, \quad(d+c)-(a+b)=0 \bmod 3
$$

Since $\delta_{\mathrm{GS}}^{2}=-30$ (see (12) ) $T_{2}$ does not have a flat potential. Nevertheless, the essential role of this field is to shift the value of $S$ by terms logarithmic in $T_{2}$. Since it is the field $y$ (defined in (2) ) which controls the value of the gauge coupling at the string scale, and not just $S_{R}$, whatever mechanism fixes the latter at realistic values must also give realistic values to $T_{2}$. For now, we will set $T_{2} \approx 1$. Henceforth, we concentrate on the moduli $T_{1}, T_{3}$ and $U_{3}$ which have rather more non-trivial potentials. We find that a numerical minimization of $V$ yields the values

$T_{1}=1.39+i(0.15+n), \quad T_{3}=0.87+i(1.50+3 m), \quad U_{3}=0.87+i(3.50+3 p)$

where in (30) $n, m$ and $p$ are arbitrary integers. In figures $1-3, V$ is plotted in the 3 moduli respectively; in each case two of the three moduli are evaluated at the minimum values given above. Fig. $1^{*}$ shows the dependence of $V$ in $T_{3}$, and shows the characteristic periodicity in $\operatorname{Im}\left(T_{3}\right)$ except that unlike the $S L(2, Z)$ case, ( as

\footnotetext{
* In this and subsequent plots, the potential $V$ has been rescaled by a factor of $-\left(24 \pi^{2} / b\right) e^{48 \pi^{2} S_{R} / b}$
} 
for example occurs for the modulus $T_{1}$ shown in Fig.2), the periodicity is $3 m$ rather than $m$. This is due to the fact that the axionic shifts in $\Gamma^{0}(3)$ are generated by $\mathcal{T}^{3}$ and not $\mathcal{T}$. The minima shown occur at the fixed points of $\Gamma^{0}(3)$. This is a consequence of taking $S_{R} \approx 2$. For larger values of $S_{R}$ (the exact value depending on the particular orbifold in question), one finds that the minima do not occur at the fixed points, the latter now being associated with saddle points. This behavior for large $S_{R}$ is easy to verify by evaluating the matrix of second derivatives of $V$ at the fixed points. The result is

$$
\begin{aligned}
\left.V_{T_{i} T_{j}}\right|_{f . p .} & =\rho_{i}\left[\delta_{i j}\left(\tilde{G}^{i, i} x(2-x)+\frac{1}{T_{i}+\bar{T}_{i}}\right)-\frac{1}{T_{j}+\bar{T}_{j}}\right] \\
\left.V_{T_{i} \bar{T}^{j}}\right|_{f . p .} & =\rho_{i}\left[\delta_{i j}\left(\left(T_{i}+\bar{T}_{i}\right)\left|\tilde{G}^{i, i}\right|^{2}-\tilde{G}^{i, i}-\frac{1+2 x-x^{2}}{T_{i}+\bar{T}_{i}}\right)+\tilde{G}^{i, i} \frac{T_{i}+\bar{T}_{i}}{T_{j}+\bar{T}_{j}}\right]
\end{aligned}
$$

where in (31), $\tilde{G}^{i, i}=\partial \tilde{G}^{i} / \partial T_{i}$ and $\rho_{i}=-|W|^{2}\left(x \prod_{j}\left(T_{j}+\bar{T}_{j}\right)\left(T_{i}+\bar{T}_{i}\right)\right)^{-1}$. Here we have only included those moduli for which $\delta_{\mathrm{GS}}^{i}$ is vanishing, since as we have seen previously, it is only for these moduli that a simple connection exists between fixed points and extrema. The eigenvalues of the matrix in (31) are $\lambda_{i}= \pm\left(x^{2}-2 x-1\right)\left(T_{i}+\bar{T}_{i}\right)^{-1} \rho_{i}$ in the large $S_{R}$ limit, so fixed points are saddle points of $V$.

Although the values of the moduli at the minimum of $V$ do depend on the value of $S_{R}$, they tend to a fairly constant limit for $S_{R} \geq 6$, given by $T_{1}=$ $1.35+i(0.43+n), \quad T_{3}=1.57+i(1.27+3 m), \quad U_{3}=1.62+i(3.33+3 p)$.

In fig. 2 we see a much 'flatter' potential for $T_{1}$ than for $T_{3}$ and $U_{3}$. Furthermore the fixed points of $S L(2, Z)$ do not correspond to extrema in this plot. This is a consequence of $\delta_{\mathrm{GS}}^{1} \neq 0$ and the fact that we have to choose $S_{R}$ away from its extremum. Indeed it should be mentioned that generally speaking, those moduli $T_{i}$ and $U$ possessing non-vanishing Green-Schwarz coefficients $\delta_{\mathrm{GS}}^{i}$ and $\tilde{\delta}_{\mathrm{GS}}^{m}$, will have their corresponding modular symmetries broken by giving $S$ an expectation value that does not correspond to an extremum. (In the present example, this affects only the $T_{1}$ modulus.) 
Finally, fig. 3 shows the dependence on the $U_{3}$ modulus which has a similar appearance to the plot in Fig. 1, except that there is a relative shift by a factor of 2 in $\operatorname{Im}\left(U_{3}\right)$. Again the periodicity of $3 p$ can be understood from the fact that the subgroup of $S L(2, Z)$ acting on $U_{3}$ (defined in (29) ), contains axionic shifts generated by $\mathcal{T}^{3}$ rather than $\mathcal{T}$. Once more the values of $U_{3}-2 i$ at the minima are also fixed points of $\Gamma^{0}(3)$.

To conclude, we have investigated the mechanism for fixing some of the moduli in orbifold compactifications due to duality invariant gaugino condensation, where the duality groups are not $S L(2, Z)$ but subgroups which are different for different moduli. We have seen that this leads naturally to anisotropic values for the moduli, in contrast to the isotropic solutions previously found in the literature. The values of those moduli for which $\delta_{\mathrm{GS}}^{i}=0$ always occur at the fixed points of the duality group for $S_{R} \approx 2$.

\section{ACKNOWLEDGEMENT}

This work is supported in part by S.E.R.C. and the work of S. Thomas is supported by the Royal Society.

\section{Table Captions}

Table. 1. Non-decomposable $Z_{N}$ orbifolds. For the point group generator $\theta$, we display $\left(\zeta_{1}, \zeta_{2}, \zeta_{3}\right)$ such that the action of $\theta$ in the complex plane orthogonal basis is $\left(e^{2 \pi i \zeta_{1}}, e^{2 \pi i \zeta_{2}}, e^{2 \pi i \zeta_{3}}\right)$.

Table. 2. Values of $C_{i m}$ and $l_{i m}$ appearing in the threshold corrections for nondecomposable orbifolds. 


\section{Figure Captions}

Fig. 1. The dependence of $V$ on the $T_{3}$ modulus for the orbifold $Z_{6}-I I-b$. Minima occur at the fixed points of the subgroup $\Gamma^{0}(3), T_{3}=\sqrt{3} / 2+i(1.5+3 m)$.

Fig. 2. The dependence of $V$ on the modulus $T_{1}$ for the orbifold $Z_{6}-I I-b$. Minima occur at $T_{1}=1.39+i(0.15+n)$.

Fig. 3. The dependence of $V$ on the $U_{3}$ modulus for the orbifold $Z_{6}-I I-b$. Minima occur at the fixed points of the subgroup $\Gamma^{0}(3)$ acting on the redefined field $U_{3}-2 i, U_{3}=\sqrt{3} / 2+i(3.5+3 p)$. 
TABLE 1

\begin{tabular}{|c|c|c|}
\hline Orbifold & Point group generator & Lattice \\
\hline$Z_{4}-a$ & $(1,1,-2) / 4$ & $S U(4) \times S U(4)$ \\
\hline$Z_{4}-b$ & $(1,1,-2) / 4$ & $S U(4) \times S O(5) \times S U(2)$ \\
\hline$Z_{6}-I I-a$ & $(2,1,-3) / 6$ & $S U(6) \times S U(2)$ \\
\hline$Z_{6}-I I-b$ & $(2,1,-3) / 6$ & $S U(3) \times S O(8)$ \\
\hline$Z_{6}-I I-c$ & $(2,1,-3) / 6$ & $S U(3) \times S O(7) \times S U(2)$ \\
\hline$Z_{8}-I I-a$ & $(1,3,-4) / 8$ & $S U(2) \times S O(10)$ \\
\hline$Z_{12}-I-a$ & $(1,-5,4) / 12$ & $E_{6}$ \\
\hline
\end{tabular}

TABLE 2

\begin{tabular}{|c|c|c|}
\hline Orbifold & $C_{i m}$ & $l_{i m}$ \\
\hline$Z_{4}-a$ & $C_{31}=2, C_{41}=2$ & $l_{31}=2, l_{41}=1$ \\
\hline$Z_{4}-b$ & $C_{31}=C_{32}=C_{41}=C_{42}=1$ & $l_{31}=l_{41}=1, l_{32}=2, l_{42}=1 / 2$ \\
\hline$Z_{6}-I I-a$ & $C_{11}=2, C_{31}=C_{32}=C_{41}=C_{42}=1$ & $l_{11}=2, l_{31}=l_{41}=1, l_{32}=3, l_{42}=1 / 3$ \\
\hline$Z_{6}-I I-b$ & $C_{11}=2, C_{31}=C_{32}=C_{41}=C_{42}=1$ & $l_{11}=l_{31}=l_{42}=1, l_{32}=l_{41}=3$ \\
\hline$Z_{6}-I I-c$ & $C_{11}=2, C_{31}=C_{32}=C_{41}=C_{42}=1$ & $l_{11}=l_{31}=l_{42}=1, l_{32}=3, l_{42}=1 / 3$ \\
\hline$Z_{8}-I I-a$ & $C_{31}=C_{32}=C_{41}=C_{42}=1$ & $l_{31}=l_{41}=1, l_{32}=2, l_{42}=1 / 2$ \\
\hline$Z_{12}-I-a$ & $C_{31}=2$ & $l_{31}=2$ \\
\hline
\end{tabular}




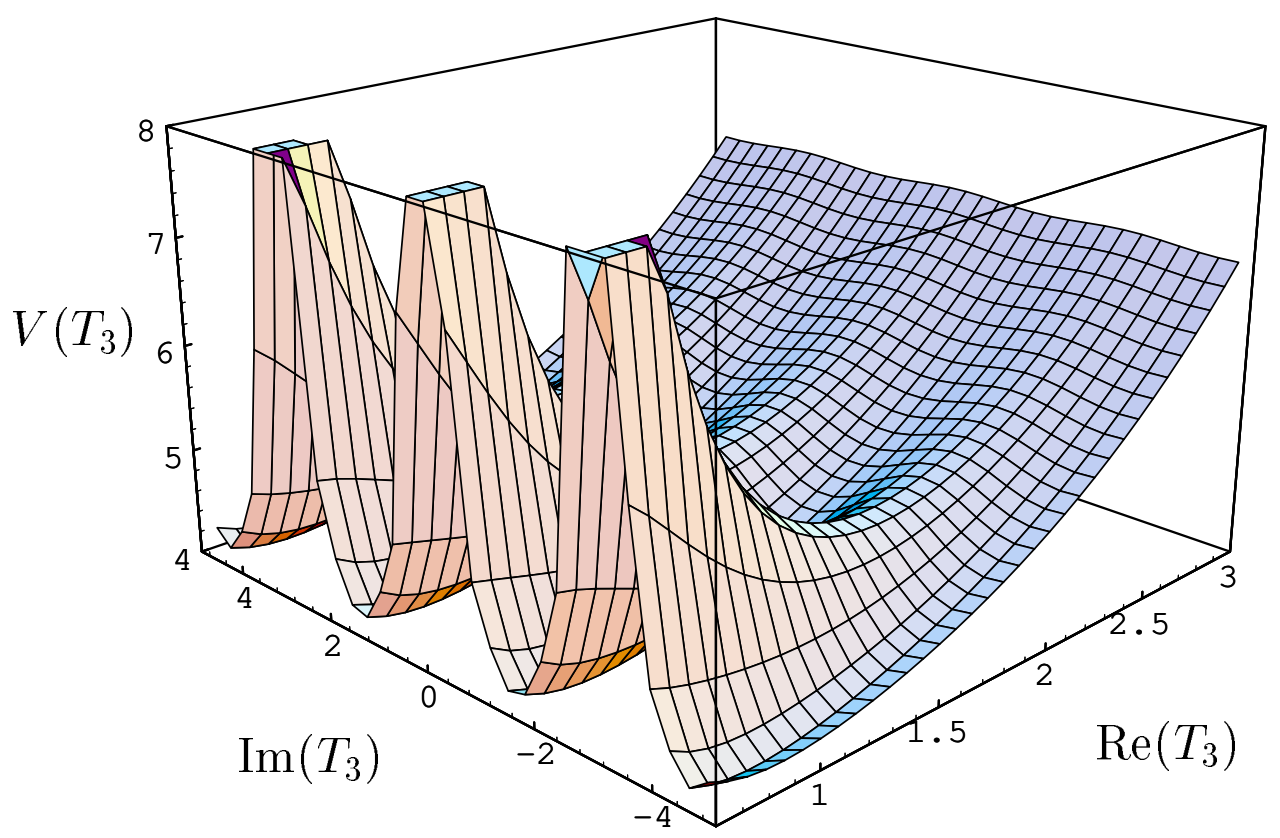

Fig1

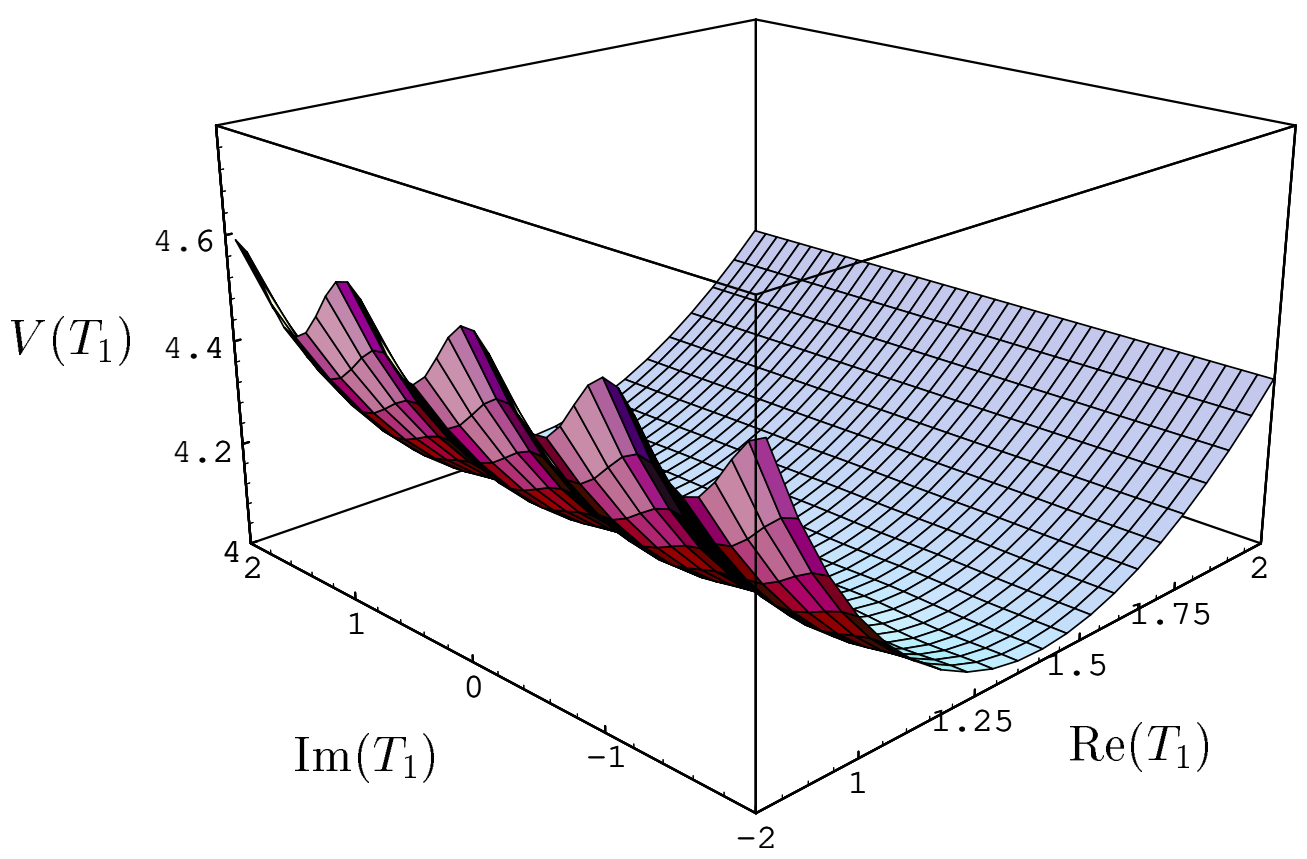

Fig2 


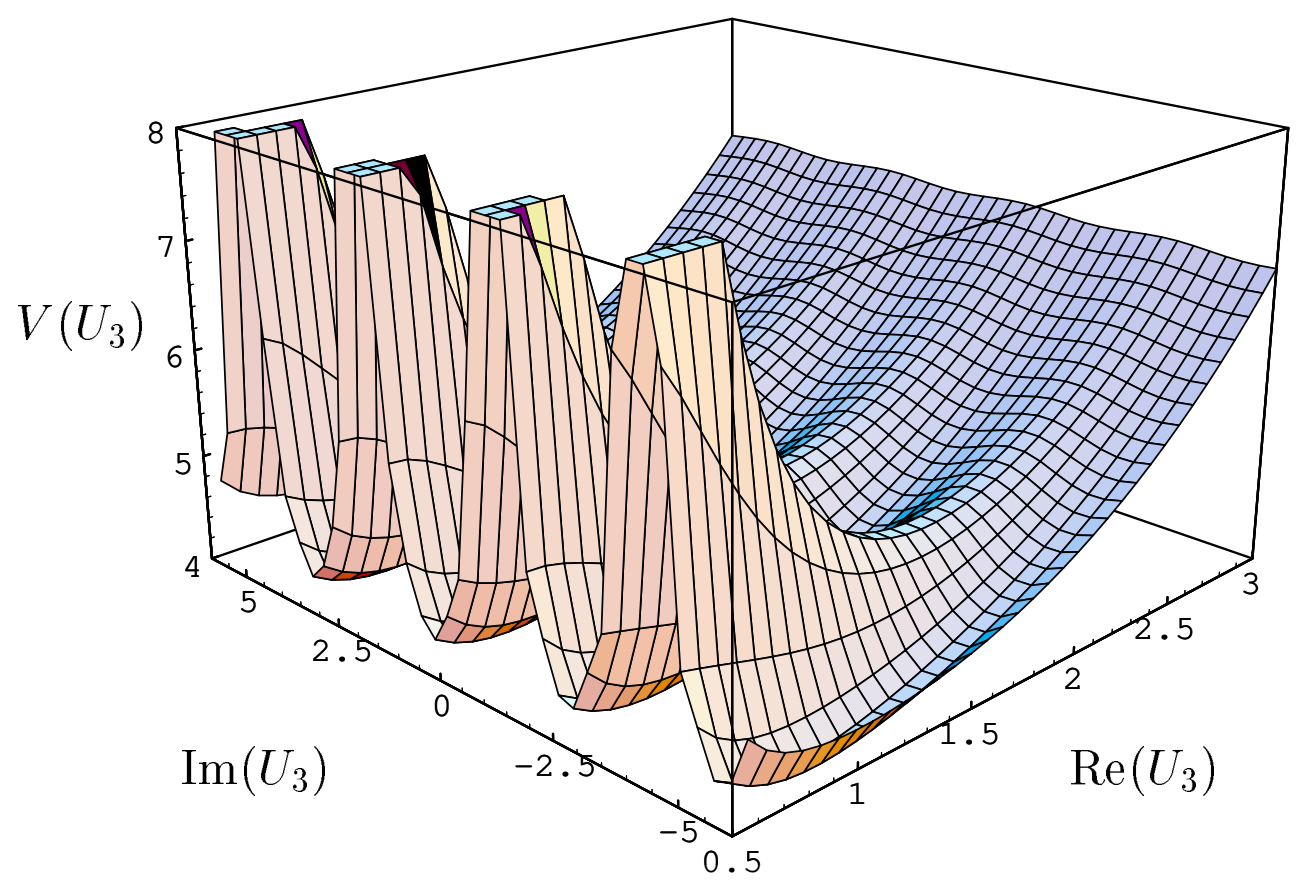

Fig3 


\section{REFERENCES}

1. L. J. Dixon, V. S. Kaplunovsky and J. Louis, Nucl. Phys. B355 (1991) 649.

2. J. P. Derendinger, S. Ferrara, C. Kounnas and F. Zwirner, Nucl. Phys. B372 (1992) 145.

3. J. Louis, SLAC-PUB-5527 (1991); G. Lopes Cardoso and B. Ovrut, Nucl. Phys. B369 (1992) 351, UPR-0481T (1991).

4. I. Antoniadis, K. S. Narain and T. R. Taylor, Phys. Lett. B267 (1991) 37.

5. M. Dine, R. Rohm, N. Seiberg and E. Witten, Phys. Lett. B156 (1985) 55; J. P. Derendinger, L. E. Ibanez and H. P. Nilles, Phys. Lett. B155 (1985) 65; H. P. Niless, Phys. Lett. B115 (1982) 193; S. Ferrara, L. Girardello and H. P. Nilles, Phys. Lett. B125 (1983) 457; C. Kounnas and M. Porrati, Phys. Lett. B191 (1987) 91.

6. L. E. Ibanez and D. Lust, Nucl. Phys. B382 (1992) 305; B. de Carlos, J. A. Casas and C. Munoz, CERN-TH6681/92 .

7. A. Font, L. E. Ibanez, D. Lust and F. Quevedo, Phys. Lett. B245 (1990) 401; S. Ferrara, N. Magnoli, T. R. Taylor and G. Veneziano, Phys.Lett. B245 (1990) 409; M. Cvetic, A. Font, L. E. Ibanez, D. Lust and F. Quevedo, Nucl. Phys. B361 (1991) 194.

8. L. Dixon, talk presented at the A. P. S. D. P. F. Meeting at Housto (1990); V. Kaplunovsky, talk presented at "Strings 90" workshop at College Station (1990); L. Dixon, V. Kaplunovsky, J. Louis and M. Peskin, unpublished.

9. J. A. Casas, Z. Lalak, C. Munoz and G. G. Ross, Nucl. Phys. B347 (1990) 243.

10. H. P. Nilles amd M. Olechowsky, Phys. Lett. B248 (1990) 268; P. Binetruy and M. K. Gaillard, Phys. Lett. B253 (1991) 119.

11. J. Louis, SLAC-PUB-5645 (1991). 
12. D. Lust and T. R. Taylor, Phys. Lett. B253 (1991) 335.

13. D. Lust and C. Munoz, Phys. Lett. B279 (1992) 272; B. de Carlos, J. A. Casas and C. Munoz, Nucl. Phys. B339 (1993) 623.

14. P. Mayr and S. Stieberger, Nucl. Phys. B407 (1993) 725.

15. D. Bailin, A. Love, W. A. Sabra and S. Thomas, Mod. Phys. Lett. A9 (1994) 67.

16. D. Bailin, A. Love, W. A. Sabra and S. Thomas, Phys. Lett. B320 (1994) 21.

17. B. de Carlos, J. A. Casas and C. Munoz, Phys. Lett.B263 (1991) 248.

18. N. V. Krasnikov, Phys. Lett. B193 (1987) 37; T. R. Taylor, Phys. Lett. B252 (1990) 59. 
This figure "fig1-1.png" is available in "png" format from: http://arxiv.org/ps/hep-th/9405031v1 
This figure "fig2-1.png" is available in "png" format from: http://arxiv.org/ps/hep-th/9405031v1 